\title{
AVALIAÇÃO DO PERFIL DOS ANTIDEPRESSIVOS E ANSIOLÍTICOS DISPENSADOS EM UMA DROGARIA:UM ESTUDO DE CAMPO $^{1}$
}

\author{
Naiara Costa Araújo- Graduanda -UNIFSA ${ }^{2}$, \\ Daniela Teresa Da Silva Carrias-Graduanda -UNIFSA ${ }^{3}$ \\ Maria Francisca Ferreira dos Santos-Graduanda -UNIFSA ${ }^{4}$ \\ Rogelma Lima de Sá- Graduanda -UNIFSA ${ }^{5}$ \\ Maria Helena Rodrigues Mesquita Brito- Docente-UNIFSA
}

\section{RESUMO}

Ansiedade e depressão são condições psiquiátricas prevalentes e altamente comórbidas em todo o mundo, e seus tratamentos são os mais pesquisados entre os transtornos mentais. 0 presente trabalho avaliou o perfil dos antidepressivos e ansiolíticos dispensados em uma drogaria de Teresina-Pi, por ter como problema a depressão em ser uma psicopatologia com etiologia complexa e que envolve diversos sintomas e a ansiedade que pode ocorrer de maneira adaptativa ou como transtorno psicológico. Este trabalho tem como objetivo, analisar os medicamentos da classe dos antidepressivos e ansiolíticos mais dispensados em uma drogaria, durante o período de janeiro a julho de 2019. Foram incluídos somente os medicamentos da classe dos antidepressivos e ansiolíticos, que estavam no período de janeiro a julho de 2019. Foram excluídos aqueles medicamentos que não fazem parte da classe dos antidepressivos e ansiolíticos e que não entraram no período proposto. Nos resultados os ISRS estão entre os antidepressivos mais prescritos e muitas vezes são o tratamento de primeira linha, enquanto o consumo de benzodiazepínicos pode estar associado com a diminuição progressiva do estresse. Depreende-se do estudo, que tem aumentado significativamente o uso dos medicamentos

\footnotetext{
${ }^{1}$ Trabalho apresentado no Congresso Brasileiro Ciência e Sociedade (CBCS 2019), promovido pelo Centro Universitário Santo Agostinho, de 03 a 05 de outubro de 2019, em Teresina-PI.

${ }^{2}$ Naiara Costa Araújo: Graduanda do curso de Farmácia-UNIFSA

E-mail.naiaraujo.farma@outlook.com

${ }^{3}$ Daniela Teresa Da Silva Carrias: Graduanda do curso de Farmácia-UNIFSA

E-mail.danny_carrias@hotmail.com

${ }^{4}$ Maria Francisca Ferreira dos Santos: Graduanda do curso de Farmácia-UNIFSA

E-mail.franala@hotmail.com

${ }^{5}$ Rogelma Lima de Sá: Graduanda do curso de Farmácia-UNIFSA E-mail.

Rogelma.24.2014@gmail.com

${ }^{6}$ Maria Helena Rodrigues Mesquita Brito: Graduanda do curso de Farmácia-UNIFSA

E-mail.Mhrmesquita@hotmail.com
} 


\section{CONQGEESOOCIENCIASSOCIEDADE \\ Inovação, Diversidaale e Sustentahilitilade}

ANAIS CBCS 2019 | 3 a 5 de outubro de 2019 | Centro Universitário Santo Agostinho - Teresina - PI

antidepressivos e ansiolíticos, e que mesmo associados com vários efeitos adversos, são considerados uma das principais tecnologias de cuidado no tratamento da depressão e ansiedade, e com o aumento da dispensação desses medicamentos nas drogarias, considera-se o cuidado e atenção com esses pacientes.

Palavras-Chave: Medicamentos. Depressão. Ansiedade.

\section{ABSTRACT}

Anxiety and depression are prevalent and highly comorbid psychiatric conditions worldwide, and their treatments are the most researched among mental disorders. The present study evaluated the profile of antidepressants and anxiolytics dispensed in a drugstore in Teresina-Pi, because it has depression as a problem, because it is a psychopathology with a complex etiology that involves several symptoms and anxiety that may occur in an adaptive manner or as psychological disorder. This study aims to analyze the antidepressant and anxiolytic drugs most dispensed in a drugstore, from January to July 2019. Only the antidepressant and anxiolytic drugs, which were from January to July 2019, were included. July 2019. Those drugs that are not part of the antidepressant and anxiolytic class and did not enter the proposed period were excluded. In the results, SSRIs are among the most prescribed antidepressants and are often the first-line treatment, while benzodiazepine consumption may be associated with progressive stress reduction. It appears from the study, which has significantly increased the use of antidepressant and anxiolytic drugs, and that even associated with various adverse effects, are considered one of the main care technologies in the treatment of depression and anxiety, and with the increased dispensation of these drugs. In drugstores, care and attention to these patients is considered.

Keywords: Medicines. Depression. Anxiety.

\section{INTRODUÇÃO}

Ansiedade e depressão são condições psiquiátricas prevalentes e altamente comórbidas em todo o mundo, e seus tratamentos são os mais pesquisados entre os 
ANAIS CBCS 2019 | 3 a 5 de outubro de 2019 | Centro Universitário Santo Agostinho - Teresina - P

transtornos mentais (KHADEMI; HAJIAHMADI; FARAMARZI, 2019). Para Leão et al (2018) aproximadamente 450 milhões de pessoas sofrem de perturbações mentais ou neurobiológicas. Destaca-se a depressão, considerada a principal causa de incapacitação e com possibilidade de se tornar a segunda maior doença até 2030. Em segundo lugar apresenta-se a ansiedade, comumente associada aos casos de depressão e que tem um efeito importante na diminuição da qualidade de vida das pessoas.

A depressão trata de uma psicopatologia com etiologia complexa e que envolve diversos sintomas, como, a diminuição da autoestima e a presença de anedonia, geralmente com perda do significado à vida. A ansiedade, por sua vez, é caracterizada por antecipação, em longo prazo, de eventos negativos, que pode ocorrer quando o indivíduo enfrenta incertezas, ameaças existenciais ou perigos potenciais/reais. A ansiedade pode ocorrer de maneira adaptativa ou como transtorno psicológico, e a severidade e o tempo de permanência desse estado determinam a diferença entre essas ocorrências. Embora haja clara distinção entre a ansiedade e a depressão, os sintomas apresentados pelos indivíduos nem sempre são característicos apenas da condição depressiva ou ansiosa, podendo ocorrer de maneira inespecífica (MARTINS, et al, 2019).

Segundo Teixeira et al (2015), a depressão é um transtorno mental de grande prevalência. Em 2012, a Organização Mundial da Saúde (OMS), estima que afetou 320 milhões de pessoas no mundo, sendo um número alarmante para uma doença não transmissível. O uso de antidepressivos têm aumentado em diversos países e os medicamentos mais consumidos foram classificados em três grupos: Inibidores Seletivos da Recaptação da Serotonina (ISRS) entre eles, citalopram, escitalopram, fluoxetina, fluvoxamina, paroxetina e sertralina; os Tricíclicos, entre eles, amitriptilina, imipramina, clomipramina, e os Inibidores Seletivos da Recaptação de Serotonina e Noradrenalina (ISRSN) como, duloxetina e venlaflaxina.

De acordo com Naloto et al (2016), o uso dos ansiolíticos tem aumentado significativamente na última década nos países como Austrália, França Espanha e Brasil, sendo o grupo dos benzodiazepínicos mais prescritos e utilizados. Os benzodiazepínicos consistem o grupo de psicotrópicos mais comumente utilizados na prática clínica, devido 


\section{conghESSO CIENCIAESOCIEDADE \\ mans}

ANAIS CBCS 2019 | 3 a 5 de outubro de 2019 | Centro Universitário Santo Agostinho - Teresina - P

as suas quatros atividades principais: ansiolítica, hipnótica, anticonvulsivante e relaxante muscular, mas em geral são indicados para os transtornos de ansiedade, insônia e epilepsia.

Há uma década, o Brasil incorporou a atenção à saúde mental às ações da Atenção Básica, garantindo panorama favorável ao atendimento aos quadros depressivos, pois possibilitou mais acesso ao tratamento do usuário com depressão. Na Atenção Básica, o atendimento à depressão é sustentado por um conjunto de políticas que possibilita construir modelo de atenção que visa ao atendimento integral do paciente. Nessa linha de raciocínio, os processos de intervenção dos profissionais exigem atuação em concepção ampliada, interagindo com os diferentes campos do conhecimento (MOTTA; MORÉ; NUNES, 2017).

Este trabalho tem como objetivo, analisar os medicamentos da classe dos antidepressivos e ansiolíticos mais dispensados em uma drogaria, durante o período de janeiro a julho de 2019.

\section{METODOLOGIA}

Esse trabalho trata-se de um estudo de campo, observacional sem intervenção descritivo, transversal e quantitativo. A pesquisa foi realizada em uma Drogaria, localizada na Rua Gilbués, №2870- Bairro São Pedro- CEP-64018010, Teresina-PI.

Foram incluídos somente os medicamentos da classe dos antidepressivos e ansiolíticos, que estavam no período de janeiro à julho de 2019. Foram excluídos aqueles medicamentos que não fazem parte da classe dos antidepressivos e ansiolíticos e que não entraram no período proposto. Os dados foram coletados através do balanço 


\section{CONGEESSOC CIENCIAESOCIEDADE

ANAIS CBCS 2019 | 3 a 5 de outubro de 2019 | Centro Universitário Santo Agostinho - Teresina - P|

trimestral na drogaria, após a assinatura do proprietário no termo de autorização do fiel depositário. A análise estatística dos dados foi realizada através do programa word, sendo os resultados expostos na forma de tabela.

\section{RESULTADOS E DISCUSSÃO}

De acordo com Costa e Martins (2018), por serem relativamente seguros e terem poucos efeitos colaterais, incluindo menor risco de efeitos anticolinérgicos e cardiovasculares, os ISRS (Inibidores Seletivos da Recaptação de Serotonina) estão entre os antidepressivos mais prescritos conforme a (tabela 1), e muitas vezes são o tratamento de primeira linha.

Segundo Pizzol et al (2019), estudos internacionais mostram que antidepressivos (Inibidores Seletivos da Recaptação de Serotonina- ISRSs) estão entre os dez fármacos mais utilizados na amamentação. Ainda para Duque et al (2017) a maioria dos estudos para o controle da ansiedade em adolescentes foi conduzido com a sertralina. Além disso, estudos foram conduzidos com outros ISRSs, como fluoxetina e fluvoxamina para múltiplos tipos de ansiedade; venlafaxina para ansiedade generalizada e paroxetina na fobia social.

Para Krause e Sredni (2016) os ISRSN ou antidepressivos duais, duloxetina e venlafaxina, em doses mais baixas agem predominantemente como os inibidores seletivos da receptação da serotonina (ISRS), em doses mais altas inibem também a recaptação da noradrenalina, seus principais efeitos adversos: náusea, sedação, constipação, xerostomia, diminuição do apetite, ansiedade, tonturas, fadiga, insônia, disfunção sexual, hiper-hidrose, hipertensão arterial e ataxia. 


\section{CONGEESSOC CIENCIAESOCIEDADE

ANAIS CBCS 2019 | 3 a 5 de outubro de 2019 | Centro Universitário Santo Agostinho - Teresina - P|

Ressalta-se que os antidepressivos tricíclicos atuam por meio da inibição da recaptação de serotonina e norepinefrina, com efeito, altamente anticolinérgico relacionado a efeitos adversos como visão turva, comprometimento da memória e aumentando o risco de alterações psicomotoras, podendo apresentar outros efeitos centrais relacionados a fraqueza e fadiga. A amitriptilina é um dos medicamentos mais populares deste grupo, (tabela 1). E a classe dos antagonistas dos receptores alfa 2 agem bloqueando a recaptação aumentando a noradrenalina e seus efeitos são, aumento do apetite, sedação porque bloqueia HI. Esse grupo causa menos efeitos colaterais que os compostos tricíclicos (BANDEIRA, et al, 2018).

Os benzodiazepínicos agem potencializando o efeito do GABA (ácido gamaaminobutírico) que é um neurotransmissor inibitório. O uso prolongado desse grupo mesmo que em baixas dosagens, é fator de risco para o desenvolvimento dos efeitos adversos que podem manifestar-se por sonolência, vertigem, cansaço, confusão mental, cefaleia, ansiedade, letargia, ataxia, hipotensão postural, amnesia retrógrada, acidentes, tolerância e dependência (NALOTO, et al, 2016).

O consumo elevado de benzodiazepínicos conforme (tabela 1), pode estar associado com a diminuição progressiva da resistência da humanidade para tolerar o estresse, com a inclusão de novas drogas, e com a pressão crescente da propaganda por parte da indústria farmacêutica. Acrescenta-se, ainda, o fato dos medicamentos serem considerados uma das principais tecnologias contemporâneas de cuidado, prometendo afastar qualquer sofrimento da sociedade atual, como depressão, ansiedade, transtornos psicóticos, solidão, crises econômicas e tristeza, apenas com a administração de uma eficaz substância química no organismo (ALVIM et al,2017). 


\section{CONGEESSOC CIENCIAESOCIEDADE \\ Inovação, Diversidaale e Sustentahilitilade}

ANAIS CBCS 2019 | 3 a 5 de outubro de 2019 | Centro Universitário Santo Agostinho - Teresina - P|

TABELA 1. Resultado da quantidade de medicamentos dispensados por caixa das classes dos antidepressivos e ansiolíticos em uma drogaria em Teresina-PI

\begin{tabular}{l|cccccccc} 
Medicamento/ Classe & Jan & Fev & Mar & Abr & Mai & Jun & Jul & Total \\
\hline Escitalopram (ISRS) & 1 & 1 & 1 & 0 & 2 & 1 & 2 & 8 \\
Sertralina (ISRS) & 0 & 0 & 4 & 1 & 2 & 1 & 0 & 8 \\
Amitriptilina (tricíclicos) & 2 & 2 & 0 & 1 & 2 & 2 & 0 & 7 \\
Fluoxetina (ISRS) & 0 & 2 & 3 & 2 & 3 & 0 & 0 & 10 \\
Venlafaxina (ISRSN) & 0 & 0 & 0 & 0 & 0 & 2 & 0 & 2 \\
Duloxetina (ISRSN) & 0 & 0 & 0 & 1 & 1 & 0 & 1 & 3 \\
Mirtazapina (Antagonista Recep alfa 2) & 0 & 0 & 0 & 0 & 0 & 1 & 0 & 1 \\
Nortriptilina (tricíclicos) & 0 & 0 & 0 & 1 & 0 & 0 & 0 & 1 \\
Trazodona (inibidor fraco Recep serotonina) & 0 & 0 & 0 & 0 & 0 & 1 & 0 & 1 \\
Clonazepam (BZDP) & 7 & 7 & 7 & 11 & 7 & 2 & 8 & 49 \\
Bromazepam (BZDP) & 0 & 2 & 0 & 2 & 6 & 0 & 0 & 10
\end{tabular}

Fonte: Word, 06/09/2019.

\section{CONSIDERAÇÕES FINAIS}

Depreende-se do estudo, que tem aumentado significativamente o uso dos medicamentos antidepressivos e ansiolíticos, e que mesmo associados com vários efeitos adversos, são considerados uma das principais tecnologias de cuidado no tratamento da depressão e ansiedade. Com o aumento da dispensação desses medicamentos nas drogarias, considera-se o cuidado e atenção com esses pacientes, sendo de extrema importância o acompanhamento farmacoterapêutico, na busca do uso racional desses medicamentos pela população.

\section{REFERÊNCIAS}

ALVIM, M.M et al. Prevalência e fatores associados ao uso de benzodiazepínicos em idosos da comunidade. Rev Bras Geriatr Gerontol. Rio de janeiro, v.20, n.4, p.463-474, 2017. 


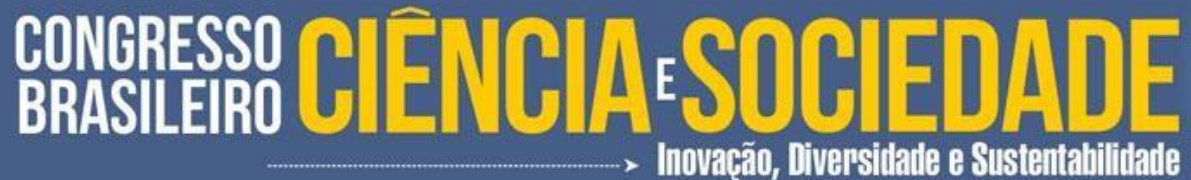

ANAIS CBCS 2019 | 3 a 5 de outubro de 2019 | Centro Universitário Santo Agostinho - Teresina - P

BANDEIRA, V. A. et al. CAntidepressant use and the components of the frailty syndrome. Rev. Bras. Geriatr. Gerontol. Rio de Janeiro, v.21, n.1, p.7-15, 2018.

COSTA, M; MARTINS, N. Hyponatremia associated with antidepressants: a Review. J. bras. psiquiatr. Rio de Janeiro, v.67, n.1, 2018.

KHADEMI, M; HAJIAHMADI, M; FARAMARZI, M. The role of long-term psychodynamic psychotherapy in improving attachment patterns, defense styles, and alexithymia in patients with depressive/anxiety disorders. Trends Psychiatry Psychother. San Diego, v.41, n.1, p.43-50, 2019.

KRAUSE, L.H; SREDNI, S. Systemic drug therapy for neuropathic pain. Rev Dor. São Paulo, V.17, n.1, p. 1-4, 2016

LEÃO, A. M. E et al. Prevalence and Factors Associated with Depression and Anxiety among University Students in the Field of Health in a Large Urban Center in the Northeast of Brazil. Revista Brasileira de Educação Médica. Fortaleza, v.42, n.4, p.5565, jul, 2018.

MARTINS, B.G. et al. Depression, Anxiety, and Stress Scale: psychometric properties and affectivity prevalence. J Bras Psiquiatr. São Paulo, v.68, n.1, p.32-41, fev, 2019.

MOTTA, C. C. L. D; MORÉ, C. L. O. O; NUNES, C. H. S. D. S. Psychological assistance provided to patients diagnosed with depression in primary care. Ciência \& Saúde Coletiva, Santa Catarina, v.22, n.3, p.911-920, 2017.

NALOTO, D. C. C. et al. Prescription of benzodiazepines for adults and older adults from a mental health clinic. Ciência \& Saúde Coletiva, São Paulo, v.21, n.4, p.12671276, 2016.

TEIXEIRA, V. et al. Utilización de antidepressivos en pacientes ambulatorios del Hospital Policial. Rev Psiquiatr Urug, Uruguai, v.79, n.1, p.9-48, jul, 2015. 